\title{
GLOBALIZACIÓN Y LA PARADOJA DE DERECHOS SOCIALES EN BRASIL*
}

\author{
GLOBALIZATION AND THE PARADOX \\ OF SOCIAL RIGHTS IN BRAZIL
}

GLOBALISATION ET LA PARADOXE

DU DROITS SOCIAUX AU BRASIL

\author{
Joaquim Carlos RACY** \\ Wagner Roberto RAMOS GARCIA JUNIOR ${ }^{* * *}$
}

\begin{abstract}
RESUMEN: El proceso de globalización es un fenómeno económico que viene del sistema capitalista que está presente en el proceso de las relaciones económicas e internacionales en varios países. Una de las características de este proceso ocurre en la mutación capitalista proporcionando innovación y la aparición de nuevas plataformas tecnológicas orientadas al consumo de bienes y servicios compartidos, tales como plataformas Uber, iFood y Airbnb. En este sentido, este comentario abordará estas características de la globalización moderna, fundamentado en economía solidaria se centra en el estudio desde la perspectiva de la innovación tecnológica y también algunos efectos externos sobre la informalidad y el impacto social que la discusión ofrece, especialmente en cuanto a la importancia de las políticas públicas internacionales que guían el debate sobre el papel de los Estados en la promoción del mínimo existencial para la población. La polarización política complica el debate sobre el verdadero papel del Estado en el análisis académico contemporáneo y la
\end{abstract} 2019.

** Doctor en historia por la Pontificia Universidad Católica de Sao Paulo.

*** Maestrando en economía y mercado por la Universidad Presbiteriana Mackenzie.

Revista Latinoamericana de Derecho Social
Núm. 30, enero-junio de 2020, pp. 125-137 
desapasionada globalizada ayuda a clarificar la preparación de los locales de la política pública y el desarrollo económico. Dados estos supuestos, la cuestión central de este artículo se basa en la siguiente pregunta: ¿cuál es el papel del Estado en un mundo globalizado?

Palabras clave: globalización, economía compartida, política pública, bienestar social del Estado.

ABSTRACT: The process of globalization is an economic phenomenon originating from the capitalist system that is present in the process of economic and international relations in the most diverse countries. One of the characteristics of this process is the mutation of capitalism, fostering innovation and the emergence of new technological platforms aimed at the consumption of shared goods and services, such as the Uber, Ifood and Airbnb platforms. In this sense, the present article will address these characteristics of modern globalization, substantiated in the Shared Economy focusing on the study from the perspective of technological innovation and also some externalities about the informality and social impacts that the discussion addresses mainly regarding the importance of international public policies which guide the debate about the actions of the States in promoting the existential minimum for the population. Political polarization hampers the debate about the true role of the state in the globalized contemporaneity and dispassionate academic analysis contributes to clarify the premises of public policymaking and economic development. In view of these premises, the guiding question of the present article is based on the following question: what is the role of the State in the globalized world?

Keywords: Globalization, Sharing Economy, Public Policies and Welfare State.

RÉSUMÉ: Le processus de globalisation c'est un phénomène économique que surgé du système capitaliste qui est présente dans le processus des relations économiques et internationales dans divers pays. Une des caractéristiques de ce processus se produit á cause de la transition capitaliste générant d'innovation et la apparition de neuf plateformes technologiques sur l'usage de biens et de services telles que Uber, Ifood et Airbnb. En ce sens, l'article abordé cette caractéristiques de la globalisation moderne, fondé au économie solidaire ce centré dans l'étude pendant la perspective d'innovation technologique et aussi certains effets externes sur la informalité et l'impact sociale qui la discussion offre, particulière- 
ment sur la importance du politiques publiques internationales que offre le débat du rôle des états dans la promotion minimum existentiel pour la société. La polarisation politique difficulté le débat sur le vrai rôle l'état dans le analyse académique contemporain et détachement globalisé aidé à classer la préparation du locales de la politique publique et le développement économique. En raison de ces hypothèses, la question centre de cet l'article se fondé dans la repose à la question suivante : quel est le rôle du état en une monde globalisé?.

SUMARIO: I. La globalización y sus definiciones múltiples. II. Estado de salud social. III. Convención Internacional de Derechos Humanos, la creación de la ONUy la política pública. IV. Externalidades y la disfunción de la política pública en Brasil. V. Responsabilidad social de las empresas y la política pública en el sector privado. VI. Final. VII. Referencias.

\section{LA GLOBALIZACIÓN Y SUS DEFINICIONES MÚLTIPLES}

$\mathrm{H}$ ay mucho desacuerdo en la literatura acerca de la definición del proceso de globalización. La discrepancia se debe a que algunos ajustes sólo se refieren a la naturaleza económica del proceso, otros sólo Al intercambio social y multidimensional, y también hay ajustes que se limitan a caracterizar el proceso de la ideología neoliberal y el sistema económico capitalista. ${ }^{1}$

A pesar de la nubosidad sobre sus definiciones, es necesario partir de la premisa de que la globalización debe ser vista como un proceso que se origina en un movimiento eminentemente económico que resulta en una mayor interacción entre los países. ${ }^{2}$ A pesar de que el proceso de integración entre los países y el intercambio social son inherentes a las características de la globalización, los conflictos entre los gobiernos, la soberanía y el libre comercio son bastante evidentes, a menudo resulta en el proteccionismo o incluso las guerras comerciales. Desde el punto de vista político, el proceso de globalización tiene influencia en diversos tratados, convenciones y acuerdos internacionales relativos principalmente a la libre circulación de los problemas

1 Campos, Louis y Canavezes, Sara, Introducción a la globalización, Bento Jesus Caraga Instituto, Departamento de la CGTP-IN, Lisboa Formación, 2007, p. 6.

2 Mordaz, Joaquim Carlos, "La globalización y la identidad nacional", Revista de Economía Mackenzie, Sao Paulo, vol. 10, núm. 1, 2012, pp. 113-120. 
de las personas en la Unión Europea (Acuerdo de Schengen) y el Mercosur (Acuerdo de Residencia).

\section{ESTADO DE SALUD SOCIAL}

La discusión sobre los límites a la intervención del Estado en la economía y el medio ambiente social ha perdurado durante siglos. La historia demuestra que el Estado ya poseía varias caras, a través del Estado liberal y absolutista, totalitario y, de forma más contemporánea, el Estado de bienestar social. El Estado de bienestar social es el sistema legal y político más ampliamente adoptado en las democracias liberales y es el modelo diseñado por la Constitución Federal de Brasil de 1988.

Art. 6 son los derechos sociales a la educación, la salud, la alimentación, el trabajo, la vivienda, el transporte, el ocio, la seguridad, la seguridad social, la maternidad y la protección de la infancia, la asistencia a los indigentes, como este Constitución (Brasil, 1988).

Las principales características del Estado de bienestar social son la fuerte intervención reguladora en el sistema económico, junto con un sistema jurídico destinado a la protección social y la distribución del ingreso a través del servicio público a necesidades básicas de los ciudadanos, como la educación, la salud y la seguridad. La definición de Estado de bienestar puede ser entendida como un conjunto de servicios y beneficios sociales de alcance universal promovidos por el Estado con el fin de garantizar una cierta "armonía" entre el avance de las fuerzas del mercado y la estabilidad social relativa, que proporciona los beneficios de la sociedad. La seguridad social significa que las personas mantengan una base material mínima y el nivel de los estándares de vida, que puede hacer frente a los efectos nocivos de la estructura de la producción capitalista desarrollada y excluyente. ${ }^{3}$ Sin embargo, mientras haya claridad y nitidez en el papel del Estado de bienestar social para intervenir en los ámbitos económico y social, el proceso de globalización encarnado por el sistema capitalista para innovar y crear nuevas tecnologías a menudo puede exceder los límites de la soberanía estatal. Como la difusión de los datos cifrados, el flujo de la comunicación privada y la apertura de los mercados

3 Gomes, Fabio Guedes, "Conflictos y el bienestar estado social: Estado y el desarrollo social en Brasil”, Rev. Adm. Pública, Río de Janeiro, 2006, p. 203. 
altamente eficientes a expensas de los servicios públicos; de alguna manera, mitigar la soberanía del Estado por el agitado proceso de destrucción creativa. En este sentido, para el día ${ }^{4}$ el desafío en el mundo globalizado es identificar el papel del Estado-nación como sus prerrogativas tanto económicas, culturales, políticas y sociales han sido mitigadas por el proceso de globalización.

\section{CONVENCIÓN INTERNACIONAL DE DERECHOS HUMANOS, LA CREACIÓN DE LA ONU Y LA POLÍTICA PÚBLICA}

A lo largo de la historia, el hombre ha enfrentado varias dificultades durante todo el proceso de la civilización. Tormentas, ventiscas, el hambre, la guerra, la enfermedad, la esclavitud y todo tipo de opresión que acompañaron y acompañan a la humanidad de hoy. Antes que el proceso de industrialización, junto con los nuevos fenómenos de la globalización y la aparición de guerras mundiales, el sistema geopolítico adoptó un nuevo sistema relacionado con el derecho de la cooperación y una política universal para proteger los derechos que garantizan la dignidad y la humanidad de todas las civilizaciones.

Desde el punto de vista histórico, “...el derecho humanitario, la Sociedad de Naciones y la Organización Internacional del Trabajo están situados como los primeros hitos en el proceso de internacionalización de los derechos humanos". ${ }^{5}$ Después de la Primera Guerra Mundial, con la intención de poner de relieve las cuestiones relacionadas con la soberanía de los estados, lo hizo la Liga de las Naciones que, de acuerdo con los dictados de la ley internacional, fue la cristalización de la derecha de la cooperación entre países.

Por desgracia, la cooperación entre los países no duró, por lo que llevó a la Segunda Guerra Mundial a ser aún más devastadora que la primera, que dio lugar a una serie de Estados totalitarios. Es importante destacar que la ONU surge en el contexto de la Segunda Guerra Mundial “...Ahora el despertar de la conciencia entre los países en los que no se respetan los derechos humanos, la convivencia pacífica entre las naciones era imposible". ${ }^{6}$

${ }_{4}$ Días, Reinaldo, Introducción a la sociología, 2a. ed., Sao Paulo, Pearson Prentice Hall, 2012, p. 213.

5 Piovesan, Flavia, Los derechos humanos y el derecho constitucional internacional, 18a. ed., Sao Paulo, Saraiva, 2018, p. 203.

6 Comparato, Fabio Konder, La afirmación histórica de los derechos humanos, 11a. ed., Sao Paulo, Saraiva, 2017, p. 226. 
En esta melodía, aunque la ONU tiene la tarea de garantizar la paz y la seguridad a nivel internacional, su propia estructura administrativa obstaculiza los principales objetivos debido a la complejidad de las relaciones internacionales, así como los intereses políticos de los miembros del Consejo de Seguridad. En lo que se refiere a la tarea de mantener la paz y la seguridad internacional, que es el primero de los propósitos y principios de la ONU, está claro que ha sido violada debido a la estructura oligárquica del Consejo de Seguridad, donde los miembros tienen derecho de veto permanente. ${ }^{7}$ Es imperativo señalar que después de los horrores del nazismo, la institucionalización de los derechos humanos demostró ser indispensable. Así como la ética desde el punto de vista legal. La Segunda Guerra Mundial dio lugar a la ruptura de estos derechos, el escenario posguerra dejó por reconstruirlas. ${ }^{8}$

El 10 de diciembre de 1948 se aprobó la Declaración Universal de los Derechos Humanos (DUDH). Es un hito en la historia de la humanidad en la que se reconoció los derechos relativos a la vida, la libertad, la propiedad y la dignidad de todo ser humano sin distinción de raza, credo o color. La Declaración Universal de 1948 pretende esbozar un orden público mundial basado en el respeto de la dignidad humana, para consagrar los valores universales básicos. Desde su preámbulo, se afirma la dignidad inherente a toda persona humana, el titular de la igualdad y los derechos inalienables. Es decir, para la Declaración Universal ser persona es el único requisito para tener derecho a los derechos. ${ }^{9}$ La DUDH forma la influencia de las relaciones geopolíticas incontrovertibles y pilares consolidados en la democracia liberal. Las relaciones sociales entre las naciones-estados, los individuos y las instituciones fueron formadas por la Declaración Universal.

\section{EXTERNALIDADES Y LA DISFUNCIÓN DE LA POLÍTICA PÚBLICA EN BRASIL}

La realidad brasileña muestra que el proceso de globalización está en pruebas en el país, especialmente con las aplicaciones tecnológicas que promueven la inventiva, numerosas instalaciones para la movilidad urbana y alternativa de ingresos para numerosas personas económicamente activas, la implementa-

\footnotetext{
7 Ibidem, p. 230.

8 Piovesan, Flavia, Los derechos humanos y el derecho constitucional internacional, 18a. ed., Sao Paulo, Saraiva, 2018, p. 232.

9 Idem.
} 
ción de políticas públicas orientadas a la calidad la vida y los derechos básicos de los ciudadanos muestran perdidas hoy en día para la población.

Según la ONG en Brasil para 2017 el 89.8\% de la población del norte no tiene acceso a la recolección y tratamiento de aguas residuales. De acuerdo con los datos que figuran en el estudio, al ritmo actual, la universalización del saneamiento básico en Brasil puede tardar más de 40 años en materializarse.

\section{FIGURA 1 \\ INDICADORES PARA EL AÑO 2017}

\begin{tabular}{|c|c|c|c|c|c|c|c|c|}
\hline \multicolumn{9}{|c|}{ Indicadores por ano - 2017} \\
\hline Localidade & $\begin{array}{l}\text { Parcela da } \\
\text { População } \\
\text { sem acesso à } \\
\text { água } \\
\text { (\% da } \\
\text { População) }\end{array}$ & $\begin{array}{l}\text { Parcela da } \\
\text { População sem } \\
\text { coleta de } \\
\text { esgoto } \\
\text { (\% da } \\
\text { População) }\end{array}$ & $\begin{array}{l}\text { Esgoto não } \\
\text { tratado } \\
\left(\text { mil m}^{3}\right)\end{array}$ & $\begin{array}{l}\text { Esgoto } \\
\text { tratado } \\
\text { sobre } \\
\text { água } \\
\text { consumida } \\
(\%)\end{array}$ & $\begin{array}{l}\text { Internações } \\
\text { totais por } \\
\text { doenças de } \\
\text { veiculação } \\
\text { hídrica } \\
\text { (Número de } \\
\text { internaçães) }\end{array}$ & $\begin{array}{l}\text { óbitos por } \\
\text { doenças de } \\
\text { veiculaçãa } \\
\text { hídrica } \\
\text { (Número de } \\
\text { óbitos) }\end{array}$ & $\begin{array}{l}\text { Renda das } \\
\text { pessoas } \\
\text { com } \\
\text { saneamento } \\
\text { (R\$ por } \\
\text { mês) }\end{array}$ & $\begin{array}{l}\text { Renda das } \\
\text { pessoas } \\
\text { sem coleta } \\
\text { de esgoto } \\
\text { (R\$ por } \\
\text { mês) }\end{array}$ \\
\hline Brasil & $16,6 \%$ & $47,6 \%$ & $5.145 .758,46$ & $45,1 \%$ & 258.826 & 2.340 & $2.533,90$ & $1.527,17$ \\
\hline $\begin{array}{l}\text { Regiẫo } \\
\text { Norte }\end{array}$ & $42,7 \%$ & $89,8 \%$ & $349.474,10$ & $17,4 \%$ & 44.984 & 198 & $2.474,68$ & $1.543,40$ \\
\hline $\begin{array}{l}\text { Regiâo } \\
\text { Nordeste }\end{array}$ & $26,8 \%$ & $73,1 \%$ & $1.102 .793,27$ & $33,5 \%$ & 124.609 & 928 & $2.004,84$ & $1.191,69$ \\
\hline $\begin{array}{l}\text { Regiâo } \\
\text { Sudeste }\end{array}$ & $8,7 \%$ & $21,4 \%$ & $2.519 .234,88$ & $51,2 \%$ & 41.904 & 752 & $2.598,27$ & $1.561,72$ \\
\hline $\begin{array}{l}\text { Regiâo } \\
\text { Sul }\end{array}$ & $10,4 \%$ & $56,1 \%$ & $793.649,33$ & $42,6 \%$ & 28.474 & 325 & $2.648,19$ & $1.904,19$ \\
\hline $\begin{array}{l}\text { Regiâo } \\
\text { Centro-Oeste }\end{array}$ & $9,9 \%$ & $46,1 \%$ & $380.606,88$ & $48,6 \%$ & 18.855 & 137 & $3.028,98$ & $1.941,43$ \\
\hline
\end{tabular}

FUENTE: ONG TRATA Brasil, 2019

Los datos son realmente alarmantes al retratar las marcas de la pobreza y la pobreza de Brasil. La aparente ausencia de una política pública choca con una curiosa paradoja, al menos, planteada por el IBGE (2017) en un estudio reciente. En Brasil, en el 92.3\% de los hogares hay por lo menos un residente que tiene un teléfono inteligente, mientras que en el $66 \%$ de los hogares del país no hay ni siquiera tratamiento de aguas residuales. ${ }^{10} \mathrm{El}$ proceso de globalización proporciona facilidad en el acceso a las herramientas tecnológicas, pero no resuelve los problemas eminentemente sociales, dado que las nuevas tecnologías no ayudan al Estado para fomentar políticas públicas de calidad de vida de los ciudadanos y la realización de los derechos constitucionales

10 Costa, Daiane, "En Brasil, el 92\% de los hogares tienen un teléfono móvil, pero sólo el 66\% han tratado de aguas residuales", El Globe, [sl], 24 de noviembre de 2017. 
positivizada. En este escenario, entendemos los factores externos o disfunciones de la globalización en un país subdesarrollado —el ciudadano tiene fácil acceso al teléfono inteligente, sin embargo no tiene saneamiento en su residencia- El debate ideológico que incluye el tema crea una serie de narrativas que distorsionan los datos y dificultan un análisis crítico del problema.

La polarización del debate político y económico sobre el papel del Estado en la economía y la eficacia de las políticas públicas dirigidas a la asistencia social debe ser examinada sin pasión y con prudencia científica. La acción del Estado para promover políticas públicas es totalmente compatible con la apertura de los mercados además de la concesión y asociaciones públicas y privadas en una mayor eficiencia de la prestación de servicios públicos. Incluyendo, cabe subrayar, que el marco regulador de Saneamiento de Brasil está sujeto al proceso de votación en el Congreso y es la esperanza de muchos brasileños en hacer cumplir los derechos y el acceso a los servicios básicos de salud y saneamiento. El papel de las instituciones y organizaciones internacionales en la propagación de las metas del milenio y los objetivos de inclusión social a través de los agentes económicos es muy beneficioso en la creación de la función del Estado en el mundo globalizado contemporáneo. Es através de los tratados internacionales y convenciones internacionales que los Estados ejercen su soberanía y se comprometen a implementar políticas públicas dirigidas al desarrollo económico y la lucha contra las desigualdades sociales.

\section{Los impactos sociales de la economía solidaria en Brasil}

De acuerdo con los últimos datos del IBGE (2019), Brasil tiene más de 12 millones de desocupados. Debido al desaliento, los ciudadanos ven en las aplicaciones de ahorro compartidos una forma de medir los ingresos y sustento. La facilidad para conectar y proporcionar servicios de manera autónoma, da lugar a las aplicaciones de transporte que se han transformado en los patrones más grandes de Brasil. Los datos de la PNAD y el IBGE (2019) indican la medida en que todas las aplicaciones de los trabajadores se reunieron en una nómina, esto sería 35 veces mayor que la nómina de la Post —el Estado brasileño es el más grande en número de empleados 109000 (Apps como UBER y iFood 2019)—. La formalización de estas aplicaciones está formada por los trabajadores que se unieron a la Junta de Comercio. Microempresarios individuales de la famosa mei ya tienen más de 8 millones de personas que utilizan las plataformas tecnológicas para garantizar su subsistencia. Sin em- 
Esta revista forma parte del acervo de la Biblioteca Jurídica Virtual del Instituto de Investigaciones Jurídicas de la UNAM http://www.juridicas.unam.mx/

bargo, la precariedad y la "uberização" es notorio en las relaciones laborales que se dejan ver en el análisis de los largos viajes a que estos trabajadores se enfrentan para sobrevivir.

\section{GRÁFICA 1}

EL CRECIMENTO DEL NÚMERO DE CELULARES EN BRASIL

\section{Crescimento do número de MEls no Brasil}

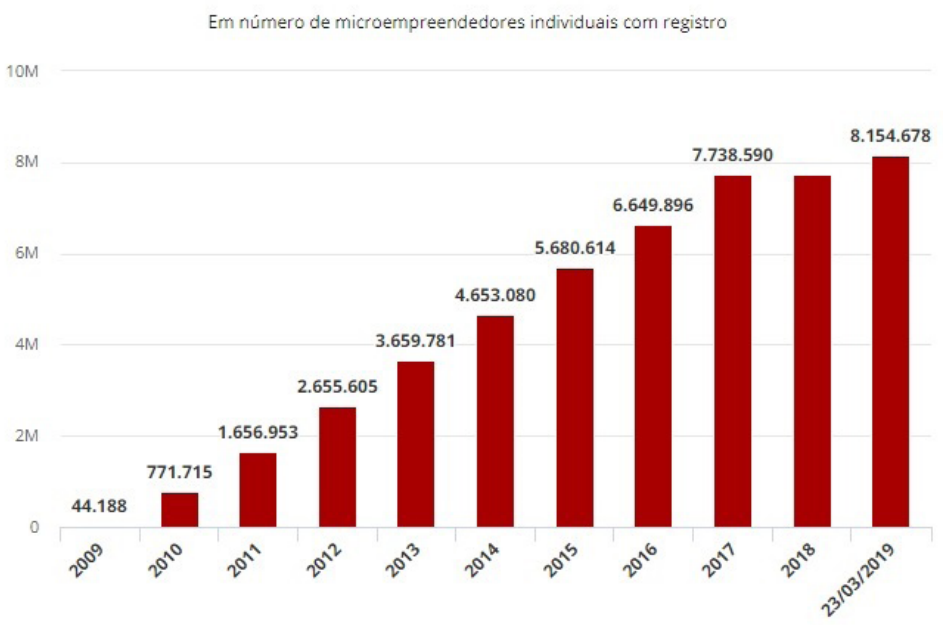

FUENTE: El portal de empresario (Alvarenga, 2019).

A pesar de toda la controversia que abarca el debate sobre la legalidad y las condiciones de trabajo que ofrecen las aplicaciones de la tecnología, el hecho es que muchas familias son apoyadas a través de estos dispositivos de ahorro compartido y su prohibición o regulación restrictiva afecta directamente a la vida de miles de personas que encontraron una manera de evitar la crisis y el desempleo persistente.

\section{RESPONSABILIDAD SOCIAL DE LAS EMPRESAS \\ Y LA POLÍTICA PÚBLICA EN EL SECTOR PRIVADO}

Ante esta perspectiva frente a la implementación de políticas públicas, derechos humanos y realización de los principios de orden social, hay nuevos temas como "Políticas Públicas y Responsabilidad Social de las organizacio- 
nes". En los últimos años han existido diversos tratados en los que se han concluido acuerdos con la intención de promover una mayor inclusión y el desarrollo económico de los países. Tanto el sector público como el sector privado prestaron atención a las medidas para promover políticas públicas que versen en el bienestar y la búsqueda de la felicidad como un amplio concepto del bien común.

En este sentido, se puede decir que las organizaciones ejercen su responsabilidad social cuando llegan a satisfacer las expectativas que los diferentes grupos de interés (grupos de interés, empleados, socios, clientes, comunidades locales, el medio ambiente, accionistas, proveedores, etcétera) tienen en su comportamiento. ${ }^{11}$ En esta melodía, inspirado principalmente por los acontecimientos históricos que se propagan a la promoción de políticas públicas en materia de derechos humanos, derechos laborales y el medio ambiente, el secretario general de la ONU en 1999 en Davos, propuso la creación de una red de grandes empresas ${ }^{12}$ que desencadenó la exhibición de los Diez Principios del Pacto Mundial inspirados en la Declaración Universal y otros tratados destinados a la dignidad humana. En cuestiones relacionadas con los derechos humanos, se establecieron dos principios:

- Principio 1. Las empresas deben apoyar y respetar la protección de los derechos fundamentales reconocidos universalmente, derechos humanos, dentro de su esfera de influencia.

- Principio 2. Las empresas deben asegurarse de que no son cómplices de violaciones de derechos humanos. ${ }^{13}$

Sin embargo, también se establecieron cuatro principios relativos a cuestiones de relaciones laborales:

- Principio 3. Las empresas deben apoyar la libertad de asociación y derecho a la negociación colectiva.

- Principio 4. Las empresas deben apoyar la eliminación de toda forma de trabajo forzoso u obligatorio.

- Principio 5. Las empresas deben apoyar la erradicación del trabajo infantil.

11 Días, Reinaldo, Sociología de las organizaciones, 2a. ed., Sao Paulo, Atlas, 2012, p. 178.

12 Días, Reinaldo, La política pública: principios, propósitos y procesos, Sao Paulo, Atlas, 2012, p. 180.

13 Idem. 
- Principio 6. Las empresas deben apoyar la eliminación de las prácticas discriminatorias en el empleo y la ocupación. ${ }^{14}$

Los otros tres principios se han centrado en la preservación y conservación del medio ambiente.

- Principio 7. Las empresas deberán mantener un enfoque preventivo que favorezca el medio ambiente.

- Principio 8. Las empresas deben fomentar las iniciativas que promuevan una mayor responsabilidad ambiental.

- Principio 9. Las empresas deben favorecer el desarrollo y la difusión de tecnologías respetuosas con el medio ambiente. ${ }^{15}$

El décimo y último principio se ocupa de la lucha contra la corrupción. "Las empresas deben trabajar contra la corrupción en todas sus formas, incluyendo la extorsión y el soborno". ${ }^{16}$ Queda, por tanto, evidente que los instrumentos de gobierno global son importantes instituciones sociales y dispositivos que permiten la creación y organización de las políticas públicas para el bienestar de la sociedad. A partir de una perspectiva sociológica de organizaciones, ${ }^{17}$ se hace hincapié en que las organizaciones se componen de personas que poseen un peso de actitudes y valores de la subjetividad. Haciendo alusión al citado acuerdo, el sociólogo sostiene que las cifras globales deben ser realizadas por las organizaciones e individuos que desean tomar un comportamiento socialmente más responsable. ${ }^{18}$

\section{FINAL}

Este trabajo aborda las definiciones acerca de la globalización y el papel del Estado-nación en el mundo globalizado. Según el estudio presentado, las nuevas tecnologías no alivian la responsabilidad de la función del Estado en la orientación e implementación de políticas públicas para la dignidad y calidad de vida. En este escenario, la aplicación de las directrices internacionales

\footnotetext{
14 Idem.

15 Idem.

16 Idem.

17 Idem.

18 Idem.
} 
como los Objetivos de Desarrollo del Milenio establecidos por la ONU, los mismos derechos humanos de la Declaración Universal son instrumentos que hacen hincapié en la soberanía de los Estados para llevar a cabo los derechos fundamentales. El debate sobre la acción del Estado en la economía con respecto a la implementación de políticas públicas no podrá referirse a los debates ideológicos. La apertura de los mercados no es condición excluyente de la política pública, sino una alternativa que permite la realización de las garantías fundamentales de los ciudadanos. Para corroborar el argumento, las empresas del sector privado han adoptado políticas públicas para el bien común a través de la responsabilidad social. Tales acciones son positivas desde el punto de vista social y no alteran la soberanía del Estado desde el punto de vista político y las relaciones internacionales.

\section{REFERENCIAS}

Alvarenga, Darlan, "País ya cuenta con 8,1 millones de microempresarios formales; ver actividades en aumento entre las mieles", G1 [sl], 3 de abril. 2019, disponible en: https://g1.globo.com/economia/noticia/2019/04/03/pais-ja-tem81-milhoes-de-microempreendedores-formais-veja-atividades-em-alta-entre-meis.ghtml.

“Apps como UBER y iFood convertirá en «el empleador» en Brasil", Examen, [sl], 28 de abril de 2019, disponible en: https:// exame.abril.com.br/economial apps-como-uber-e-ifood-sao-fonte-de-renda-de-quase-4-milhoes-de-pessoas/.

Brasil. Constitución de la República Federal de Brasil 1988. Boletín Oficial, Brasilia, DF, 5 de octubre de 1988, disponible en: http:/ / wmw.planalto.gov.br/ ccivil_03/constituicao/constituicao.btm.

CAMPOS, Louis y CANAVEzES, Sara, Introducción a la globalización, Bento Jesus Caraga Instituto, Departamento de la CGTP-IN, Lisboa Formación, abril de 2007, disponible en: https://dspace.uevora.pt/rdpc/bitstream/10174/2468/1/Introdu\%C3\%A7\%C3\%A30\%20\%C3\%A0\%20Glo baliza $\%$ C3\% $A 7 \%$ C3\% 33 o.pdf.

Comparato, Fabio Konder, La afirmación histórica de los derechos humanos, 11a. ed., Sao Paulo, Saraiva, 2017.

CostA, Daiane, "En Brasil, el 92\% de los hogares tienen un teléfono móvil, pero sólo el 66\% han tratado de aguas residuales", El globo, [SL], 24 de noviembre de 2017, disponible en: https://oglobo.globo.com/economia/no-brasil92-dos-lares-tem-celular-mas-apenas-66-tem-esgoto-tratado-22108160. 
DíAs, Reinaldo, Introducción a la sociología, 2a. ed., Sao Paulo, Pearson Prentice Hall, 2012.

DÍAS, Reinaldo, La política pública: principios, propósitos y procesos, Sao Paulo, Atlas, 2012.

DíAs, Reinaldo, Sociología de las organizaciones, 2a. ed., Sao Paulo, Atlas, 2012.

GOMES, Fabio Guedes, "Conflictos y el bienestar estado social: Estado y el desarrollo social en Brasil”, Rev. Adm. Pública, Río de Janeiro, vol. 40, núm. 2, abril de 2006, disponible en: http:/ / www.scielo.br/scielo.php?script $=$ sci_ arttext\&oid $=$ S0034-76122006000200003\&lng=en\& $\mathrm{nrm}=i$ so.

Panel de Reorganización Brasil. Indicadores. Instituto Trata Brasil, [sl], 2017, disponible en: https:// wmw.painelsaneamento.org.br/explore/ano?SE \% 5Ba\% $5 \mathrm{D}$ $=2017 \& 5 E \% 5 B 0 \% 5 D=a$ (fecha de consulta: 20 de junio de 2019).

PiOVESAN, Flavia, Los derechos bumanos y el derecho constitucional internacional, 18a. ed., Sao Paulo, Saraiva, 2018.

MORDAZ, Joaquim Carlos, "La globalización y la identidad nacional", Revista de Economía Mackenzie, Sao Paulo, vol. 10, núm. 1, 2012, disponible en: http:// editorarevistas.mackenzie.br/index.php/ rem/article / view/5176/3935 (fecha de consulta: 20 de junio de 2019). 